\section{Intensity-modulated radiotherapy for early glottic cancer: transition to a new standard of care?}

\author{
Michael A Samuels ${ }^{* 1}$, Laura M Freedman' \& Nagy Elsayyad ${ }^{1}$
}

For decades, the standard of care for radiation treatment of early larynx cancers has been conventional treatment using opposed lateral fields encompassing the larynx and overlying neck structures, including the adjacent carotid arteries. While intensity-modulated radiotherapy (IMRT) has replaced conventional radiotherapy for all other head/neck cancer situations, the use of IMRT to treat early glottic cancers remains controversial. The article reviews the published experience with IMRT for this clinical situation and provides a detailed review of the literature on radiation-induced carotid toxicity and how it might apply to the controversy. Finally, we discuss whether the radiation oncology community should transition to IMRT as a new standard of care for the treatment of early glottic cancers.

First draft submitted: 27 March 2016; Accepted for publication: 21 June 2016; Published online: 9 August 2016

\section{Development of intensity-modulated radiation therapy}

Intensity-modulated radiation therapy (IMRT) was conceived in the early 1980s through the seminal work of Anders Brahme and developed progressively by radiation physicists in the university setting through the late 1990s [1]. The first clinical IMRT treatment was delivered at Baylor University in Houston in 1994. Most major radiation equipment manufacturers offered early IMRT planning and delivery systems beginning in the late 1990s and the technique was quickly adopted into clinical practice. Over the next several years, the technique was refined progressively. IMRT revolutionized the delivery of radiation therapy by allowing the creation of radiation dose distributions into concave shapes [2]. These distributions substantially reduced dose to sensitive nearby normal structures including salivary glands, brainstem and spinal cord [3-5]. The prior technique, 3D conformal radiotherapy (3DCRT), utilized computed tomography-based planning and radiation beams from multiple angles to produce complex dose distributions, but always fundamentally convex. Therefore, the conformality created was inferior to IMRT plans.

One of the earliest applications of this technology was treatment of advanced head and neck cancers. The rationale was that traditional 3DCRT mainly utilized opposed lateral radiation beams that encompassed the tumor and draining neck lymph nodes. However, this technique simultaneously delivered the prescribed tumor dose to adjacent sensitive structures such as the parotid and submandibular salivary glands, the structures involved in swallowing, and the mandible. Severe late effects were common, including grade 3 xerostomia [6], permanent dysphagia [7] and osteoradionecrosis of the mandible [8]. Despite the use of multiple-beam techniques and sequential boost fields, the plans could only reduce dose to normal structures modestly, based on the fundamental limitations of 3D conformal planning and delivery.

'Department of Radiation Oncology, University of Miami/Sylvester Comprehensive Cancer Center, Miami, FL, USA

*Author for correspondence: msamuels2@med.miami.edu

\section{KEYWORDS}

- carotid injury

- cerebrovascular • glottis

- IMRT • intima • larynx

- radiation • stroke 
IMRT clearly presented a major dosimetric advantage over 3DCRT. To understand this, one must understand that a modern radiation treatment unit, such as a linear accelerator, produces a beam of high-energy $\mathrm{x}$-rays that exits the gantry of the unit aimed at a patient lying on a treatment couch. In years past, the beam was altered into the necessary shape, or collimated, by molded lead-bismuth alloy 'blocks' that were attached to a plastic tray and slid onto a bracket from which the beam exited the machine. This relatively crude technology was replaced in the past 15 years with an electromechanical beamshaping system called a multileaf collimator (MLC). The MLC utilizes a large number of metal rods, or 'leaves', which are programmed to move into position according to a prescribed plan to provide the necessary beam collimation without the need to mold blocks from hazardous materials. Therefore, the introduction of the MLC allowed 3DCRT to be applied more quickly and safely than previous systems [9].

IMRT took radiation treatment planning and delivery to the next level by combining two key technologies: first, it utilizes movement of the MLC during the actual beam-on time to modulate, or alter, the radiation beam as it leaves the radiation treatment unit on the way to the patient. This was an entirely novel concept. It is this beam modulation that allows the creation of concave dose distributions. However, determining the degree and timing of each movement of each leaf of the MLC is beyond the capacity of a trained physicist or dosimetrist (radiation planning staff member) to calculate and prescribe. Therefore, IMRT incorporated a shift from 'forward planning' to 'inverse planning'. Forward planning involves the dosimetrist and radiation oncologist working together to create a radiation beam arrangement in the planning computer system which overlays on a CT scan image in the region of the patient's tumor or target area. The dosimetrist then adjusts the beam arrangement as necessary to encompass the target properly at all levels while trying to avoid exposing sensitive nearby normal structures to excessive radiation dose. The plan would undergo review by the radiation oncologist and radiation physicist with possible additional adjustments in an iterative process. Inverse planning, in contrast, requires the radiation oncologist to outline (contour) each target volume and sensitive normal structure on every slice of the planning CT scan. The radiation oncologist then assigns minimum and maximum required dose levels to the target structures as well as maximum dose limits to the normal and avoidance structures. The dosimetrist enters these values into the radiation planning system along with information on the relative priority of each structure's dose values. The computer system then calculates an IMRT plan incorporating several beams (or, alternatively, a moving arc arrangement) with movement of the MLC to create a plan that achieves the radiation dosing goals set forth by the radiation oncologist. It is the combination of the physical properties of beam intensity modulation and the power of modern computing that allows the radiation oncologist to 'paint' radiation dose across the volume of interest.

IMRT is now the standard of care for treating all head and neck cancer sites in both the definitive and postoperative settings $[10,11]$. However, treatment of early glottic cancers (T1-2N0) has remained an exception. The stated rationale is that standard 3DCRT for these cases uses small opposed lateral radiation beams encompassing the larynx while sparing the spinal cord. The complexity of planning IMRT as well as the expense of the planning and delivery process is not justified with a paucity of sensitive structures to spare with IMRT [12]. The spinal cord is not included in the 3D fields, so the only critical structures to avoid would be the carotid arteries. A secondary objection to IMRT focused on the larynx with relative sparing of the carotid arteries is that it might increase the risk of a 'marginal miss.' This is a failure to treat the tumor with adequate margins to an adequate dose due to mistargeting or organ motion [13]. The converse argument and rationale for sparing the carotids is that minimizing radiation would reduce premature progression of arterial stenosis and possibly decrease the risk of premature cerebrovascular events (CVEs) associated with this progression. Since the carotid arteries are considered, radiobiologically speaking, normal structures where the functional subunits are organized 'in-series' rather than 'in-parallel', prevention of narrowing within the carotid lumen in even a short irradiated segment would be important in preventing dysfunction of the structure - that is, stroke.

\section{$2 \mathrm{D}$ radiotherapy era}

$2 \mathrm{D}$ radiotherapy for early glottic cancers was the standard of care from the 1950s until the advent of 3D planning in the 1990s. Planning typically involved a plain lateral radiograph of the neck 
in either the supine or lateral decubitus position. An external contour of the neck was obtained at the level of the vocal cords in order to determine the slope of the curvature of the neck. Field borders included the inferior aspect of the hyoid bone superiorly, the lower edge of the cricoid cartilage inferiorly and the anterior border of the vertebral bodies posteriorly. Anteriorly, the fields would extend $1 \mathrm{~cm}$ beyond the skin overlying the anterior aspect of the thyroid cartilage. This typically resulted in field dimensions of $4 \times 4$ to $5 \times 5 \mathrm{~cm}$ [13]. The neck shape as indicated by the external neck contour determined the degree of 'wedging' required to create a homogeneous dose distribution throughout the treatment volume. Wedging is a technique in which metal tissue compensators, shaped similarly to a wedge of cheese or a doorstop, were inserted into the lateral treatment beams. Wedges attenuate the radiation dose to the anterior, more narrow, portion of the neck contour to avoid giving too much radiation dose to this region, thereby minimizing excessive skin toxicity [14]. Wedges were available in several angular measurements (typically 15, 30, 45 and $60^{\circ}$ ). Use of the proper wedge allowed treatment to the larynx with a relatively homogeneous dose distribution. Treatment planning was accomplished initially using manual calculation techniques and later with computerized radiation planning systems.

Treatment beams typically included Cobalt- 60 or a low-energy X-ray beam generated by a linear accelerator. Fractionation ranged from 1.8 to 2.25 Gy per day, 5 days per week. The most common daily fraction size was $2.0 \mathrm{~Gy}$, with a total delivered dose of 66 Gy in 33 treatments. Mendenhall and colleagues from the University of Florida published a series of influential reviews of their experience with radiotherapy of early glottic cancers [13,15-17]. Their favored fractionation usually exceeded $2 \mathrm{~Gy}$, often using 2.25 Gy to a total of $63 \mathrm{~Gy}$ in 28 fractions. An anterior 'boost' field was often employed for T1a tumors.

\section{$3 \mathrm{D}$ radiotherapy era}

$3 \mathrm{D}$ radiotherapy for early glottic cancers is not substantially different from $2 \mathrm{D}$ techniques. It utilizes a noncontrasted neck CT scan in the treatment position in place of a lateral planning radiograph. The external contour of the neck is visible on the scan and no manual method of obtaining an external contour is necessary. However, the field arrangement, borders, field dimensions, wedging and dose distribution are similar regardless of whether 2D or 3D planning is used (Figure 1).

\section{Outcomes of conventional radiotherapy for early glottic cancers}

The relative simplicity of the 3D technique coupled with excellent outcomes were exemplified in Mendenhall's 1988 review of the University of Florida experience [15]. Three hundred four patients were treated from 1964 to 1984 , with a minimum 2-year follow-up. Local control for T1 lesions was $93 \%$, with $75 \%$ for T2 lesions. Ultimate local control was higher due to the availability of surgical salvage using hemilaryngectomy or total laryngectomy. Major complications were very low at $1.6 \%$. These complications included chondronecrosis, fat necrosis and chronic laryngeal edema requiring tracheostomy. This publication included a perceptive analysis of local control in relation to daily fraction size, with a review of other published data contrasting control with fractions as low as $1.8 \mathrm{~Gy}$ and as high as $2.55 \mathrm{~Gy}$. The authors concluded that optimal control and toxicity were achieved with a fraction size of 2.25 Gy. Schwaibold published a retrospective analysis of 600 patients in 1988 focusing on fraction size [18]. All were treated with conventional techniques. He concluded that a fraction size of at least 2.0 Gy was required for optimal control. The largest series of patients treated with conventional radiotherapy for T1-T2 glottic cancer was published

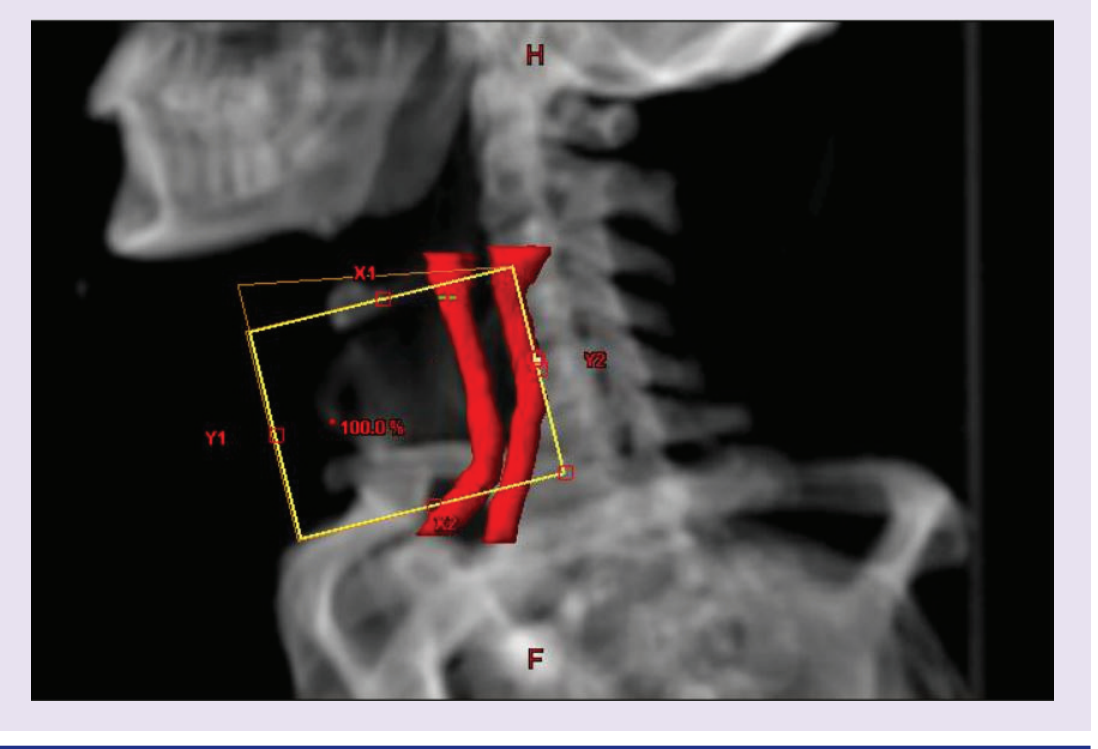

Figure 1. Sagittal view of a typical conventional radiation field. The carotid arteries are visible in red. 
by Al-Mamgani in 2013 [19]. A total of 1050 patients was treated using either conventional or accelerated fractionation (66 Gy using 2.0 Gy fractions delivered either five- or six-times per week) was evaluated. Local control was $85 \%$ at 5 years, with higher local control in the accelerated fractionation group compared with the conventional group ( 87 vs $81 \% ; p=0.006$ ). The Voice Handicap Index was measured for many of the patients. Factors associated with poor voice outcome included T2b tumor stage and continued smoking.

\section{Radiation dose fractionation: randomized} data

Optimal dose fractionation schedule of radiotherapy to treat early glottic cancers has been subject to investigation. In a randomized study, Yamazaki et al. [20] compared a conventionally fractionated course of delivering 2 Gy per fraction with a hypofractionated course delivering 2.25 Gy per fraction. One hundred and eighty patients were randomized. Total doses in the conventional arm were $60 \mathrm{~Gy}$ (minimal disease) or $66 \mathrm{~Gy}$ and those in the hypofractionated arm were 56.25 and 63 Gy. Treatment was delivered using $4 \mathrm{MV}$-ray beams in the supine position with wedged lateral radiation fields (3DCRT). At a median follow-up of 64 months, local control was $77 \%$ in the conventional arm and $92 \%$ in the hypofractionated $\operatorname{arm}(\mathrm{p}=0.004)$. No severe late complications were observed. Although local control in the conventional arm was strikingly poor, the fact that this was (and remains) the only large randomized study addressing the effect of fraction size (dose per fraction) on local control of early glottic cancers has been used by many institutions worldwide as evidence of the superiority of the hypofractionated approach.

Limitations of conventional radiotherapy

Despite high local control rates and low rates of major complications, there are inherent limitations to conventional radiotherapy for early glottic cancer. Chief among these is that the opposed lateral treatment approach (with 3DCRT or 2D) delivers a dose to the adjacent normal structures essentially the same as that delivered to the targeted portion of the larynx. Consequently, the common carotid arteries, the carotid bulbs, the proximal initial portion of the internal carotid arteries and portions of the thyroid gland receive unnecessary radiation exposure (Figure 2). This remains true, at least for the ipsilateral carotid system, even if an anterior boost field is added to the lateral treatment fields. Usually only a small volume of the thyroid gland is irradiated and thyroid insufficiency, if it occurs, is easily treated with thyroid replacement medication. However, there is a known correlation between radiation dose to the carotid system and carotid toxicity, including stenosis and accelerated progression of intima-medial thickening, both of which are positively correlated with CVEs [21]. This concern may create an incentive to avoid radiotherapy for early larynx cancer in favor of conservative surgical techniques such as vocal cord stripping or laser cordectomy. An alternative approach is to utilize a radiotherapeutic technique such as IMRT that can reduce the carotid dose.

\section{Feasibility \& dosimetric parameters of IMRT for early glottic cancers}

Evidence of the feasibility of dose reduction to the carotid arterial system at the level of the larynx utilizing IMRT in early glottic cancers is abundant. Chera performed a retrospective analysis in which five patients treated with conventional radiotherapy were replanned with IMRT for dosimetric comparison [13]. For a bilateral vocal cord plan, the mean dose to the carotids was 38 Gy with the conventional plan and 10 Gy with the IMRT plan. Osman examined a group of ten patients with Tla disease and retrospectively replanned the cases with 3DCRT, IMRT coplanar and IMRT noncoplanar methods for single vocal cord irradiation (SVCI) [22]. Coverage of the involved vocal cords and relative sparing of the carotids and uninvolved vocal cord was feasible with IMRT (both coplanar and noncoplanar approaches). There was no advantage to the noncoplanar plan. The authors advocated IMRT for this reason and posited that it would improve safety of re-irradiation in situations of recurrent disease. The same group published a technical analysis of the same dataset evaluating the accuracy and precision of daily IMRT for treatment of early glottic cancers [23]. Their analysis showed that radiation field margins around the radiation targets could be as small as $2 \mathrm{~mm}$ as long as daily image-guided radiotherapy using cone beam computed tomography was utilized. Riegel presented a group of 11 patients with T1-2 glottic cancer planned with 3DCRT, static IMRT, $180^{\circ}$ intensity-modulated arc therapy (IMAT) and $360^{\circ}$ IMAT [24]. Building on existing literature, 




Figure 2. Comparative radiation dose distributions comparing conventional radiotherapy (left) and intensity-modulated radiotherapy (right). The colorwash display indicates higher dose in red and lower dose in blue. The carotids are indicated with black arrows and the vocal cord tumor with dark red arrows.

the authors advocated volumetric tolerance doses of a mean of $35 \mathrm{~Gy}$ and a maximum of 50 Gy to these vascular structures to minimize the risk of carotid stenosis. Static IMRT and $360^{\circ}$ IMAT were both optimal (IMRT for low noncarotid normal tissue dose and good carotid sparing; $360^{\circ}$ IMAT for higher normal tissue dose but optimal carotid sparing). Matthiesen evaluated ten patients retrospectively selected for replanning with 3D, IMRT, IMAT and proton beam RT [25]. All techniques provided good target coverage. IMAT and protons were superior to 3DCRT and IMRT for mean carotid dose, with IMAT slightly better than protons (mean dose of 23 vs 24 Gy).

All of the authors noted above emphasized the dosimetric advantages of IMRT in terms of significant sparing of carotids at the level of the treated larynx (Figure 3). However, the same authors consistently noted disadvantages of IMRT in this setting compared with conventional $R T$. These disadvantages include increased planning and delivery time and expense, increased dose to normal tissues away from the carotids including the spinal cord (although within accepted tolerance limits) and the possibility of increased dose inhomogeneity that might include 'hot spots' within sensitive portions of the larynx. Additionally concerning was the possibility of missing tumor (marginal miss) from planning that focuses the high-dose radiation volume over a much smaller volume than previously utilized in conventional RT. These concerns generally precluded the authors from recommending a transition to IMRT as a new standard of care for treatment of early glottic cancers [26].
These objections merit examination. Feigenberg, in a 2007 editorial [27], cautioned against the use of IMRT in this setting. The editorial was motivated by the experience of the author and his colleagues in evaluating "several patients for laryngectomy for radiation failures in early-stage glottic cancer who were treated with IMRT." He voiced his concern that marginal misses, particularly for tumors involving the anterior commissure, could be particularly prevalent with IMRT planning. He commented that IMRT took approximately $20 \mathrm{~min}$ to deliver, compared with just a few minutes for conventional RT. The difference in time of treatment delivery led to concerns that laryngeal movement with swallowing during this prolonged treatment delivery time might reduce target dose. However, the editorial was published in 2007, when IMRT treatment times were much longer than are typical today (2-5 $\mathrm{min})[28,29]$. He also noted increased volumes of normal tissues outside of the laryngeal target to low dose exposure (referred to commonly as 'integral dose') with a theoretical increase in the risk of secondary, radiation-induced malignancies in such tissues. Feigenberg reasoned that the rate of major complications recorded in the large series from the University of Florida ranges from 1 to $2 \%$, and it was unlikely that IMRT could reduce this complication rate further. However, neither the Florida articles nor Feigenberg's editorial addressed the possibility of late vascular complications from conventional radiotherapy. Since the authors were not able to quantitate the rate of local relapse with IMRT versus conventional RT and were only citing personal experience, it is not possible for the reader to determine 




Figure 3. Comparative dose-volume histogram of radiation dose to the target volumes (magenta lines) and carotid arteries (blue lines) for conventional radiotherapy (orange arrows) and intensity-modulated radiotherapy (blue arrows). The figure demonstrates equivalent target dose with significant carotid sparing for intensity-modulated radiotherapy.

whether the recurrences seen by Feigenberg's team represented the $10-20 \%$ expected failure rate with any radiation technique, or a higher rate associated with IMRT.

Kubicek [30] noted that IMRT for all head and neck cancers resulted in an increase in an increase of the volume of nontargeted normal tissues receiving low doses of radiation (collectively called 'integral dose'). Fortunately, second malignancies from radiation therapy are so rare that it is very difficult to quantitate the degree of increased risk from this phenomenon when treating relatively small volumes as is the case with early glottis cancers.

Zumsteg [26] reported an increase in total volume of regions of high dose ('hot spots') within the targeted larynx with IMRT in early cancers of the glottis but did not quantify this finding. Matthiesen's analysis of 3D conformal RT versus IMRT versus proton RT did show a statistically significant difference in minimum and maximum doses between IMRT and 3DCRT, with more inhomogeneity seen in the IMRT group [25]. However, the absolute difference in maximum dose was $66.80 \mathrm{~Gy}$ for 3D compared with 70.12 and 69.55 Gy for IMRT and IMAT, respectively. As no volumetric data were presented, it is unknown whether these dose maxima represented hot spots large enough to be clinically significant. Furthermore, the clinically relevant, yet unanswered, question is where exactly within the targeted volume do are such hot spots located and whether their geographic distribution within the larynx is more or less predictable with IMRT compared with 3DCRT. As far as toxicity of clinical relevance is concerned, the occurrence of hot spots in air or tumor may be less clinically significant (or even desirable in case of the latter), than their occurrence in the arytenoids or the interarytenoid region, for example. Such important details have yet to be fully studied.

\section{Clinical experience with IMRT for early glottic cancers}

The first publication of results from patients treated with IMRT for early glottic cancers came from Penagaricano at the University of Arkansas in 2004 [31]. He presented three cases of T2N0M0 stage II disease. Conventional plans were created retrospectively for comparison. There was no effort in the IMRT planning process to spare the carotids and only limited follow-up was available. Toxicity was modest and resolved fully after treatment. The authors noted that IMRT reduced the high-dose volume and successfully excluded some normal tissue from this volume compared with conventional treatment.

Rosenthal published in 2010 a series of 11 patients treated with IMRT at the MD Anderson 
Cancer Center [32]. The investigators used volumetric indices of carotid dose that have since become widely adopted in evaluating IMRT plans: V35 (the percentage of the carotid within the superior and inferior extent of the planning target volume receiving 35 Gy or higher), V50 and V63. Conventional plans were provided for these same patients for comparison. Carotid doses were significantly lower with the IMRT plans where the V35, V50 and V63 were 2\%, $0 \%$ and $0 \%$ versus $100 \%, 100 \%$ and $69 \%$ in 3DCRT plans, respectively. The authors noted that treatment planning and delivery times were similar for IMRT and conventional RT. No specifics were provided relating to patient outcomes, except for a statement that "treatment results have been excellent thus far."

Berwouts provides a study from 2015 from Ghent in which 40 patients with T1-T2 disease were treated with IMRT and compared with 81 historical controls [33]. Follow-up was 3.8 years in the IMRT group and 9.0 years in the conventional group. There were no significant differences in 5-year actuarial local control between the groups, at $83 \%$ for IMRT and $74 \%$ for conventional RT $(p=0.64)$. There was significantly less skin toxicity in the IMRT group. Two patients in the IMRT group had late grade 3 hoarseness (5\%).

Zumsteg at Memorial Sloan-Kettering Cancer Center published the largest series of patients treated with IMRT for early glottic cancers in 2015 [26]. A total of 48 patients with T1-T2 disease was treated with IMRT and compared, retrospectively, with 282 patients treated conventionally. Median follow-up was 44 months for the IMRT group and 66 months for the conventional group. The median maximum dose to the planning target volume was only $105 \%$, indicating the potential for excellent dose homogeneity with IMRT. There were no significant differences in any measure of tumor control between the techniques, with 3-year actuarial local control at $88 \%$ for IMRT and $89 \%$ for conventional RT. There was no mention of toxicity. Similar to the group from MD Anderson, the authors of this series noted no major differences in time required to plan or treat patients with IMRT compared with conventional RT. These data showing equivalent rates of local control for IMRT and 3DCRT for early glottic cancers parallel the data for IMRT compared with 3DCRT in other head/neck cancer sites [5,34-35].
Al-Mamgani presented a series of 30 patients treated with IMRT to the ipsilateral larynx for SVCI for T1a disease [36]. 2-year local control was $100 \%$. A pronounced hypofractionation regimen was used $(3.63 \mathrm{~Gy} /$ fraction $\times 16$ fractions) and rates of acute and late toxicity were low. Mean maximum doses to the ipsilateral and contralateral carotids were specified in the paper (18.1 and 10.5 Gy), showing excellent carotid sparing. There was no quantitative discussion of carotid sparing for SVCI compared with bilateral larynx IMRT, but the authors did mention that the dose to both carotids was substantially lower for SVCI patients compared with previous patients treated with bilateral larynx IMRT.

\section{Radiotherapy-related carotid injury: ultrasound evaluation of intima-medial thickness}

Several imaging parameters of the carotid arteries have been studied and found to be useful predictors for ischemic strokes in asymptomatic patients including intima-media thickness [37], arterial stiffness, degree of carotid stenosis, plaque morphology and plaque total area. Radiotherapy-associated injury to carotid arteries including luminal narrowing and atherosclerosis is a risk factor for carotid ischemic events [38]. The mechanism of radiation-induced carotid injury is not entirely clear, but it is felt to be related to radiation damage to all three layers of the vessel wall, the tunica intima, tunica media and tunica adventitia [39]. The most prevalent noninvasive technique for evaluation of the carotid artery utilizes ultrasound. Ultrasound allows measurement of each individual component of vessel wall thickness and can also reveal the presence and thickness of atherosclerotic plaques [36]. An early study of carotid ultrasound evaluation of patients irradiated to the neck was undertaken by Lam and published in 2001 [40]. The cohort consisted of 71 patients treated with radiation for nasopharyngeal cancer. The control group was 51 patients newly diagnosed with the same disease before radiotherapy. Risk factors for cerebrovascular disease were the same in both groups. However, carotid stenosis was seen in 56/71 patients $(79 \%)$ in the irradiated group compared with $11 / 51(22 \%)$ in the control group. Significant stenoses of more than $50 \%$ were seen only in the irradiated group. The authors concluded that radiation treatment to the neck can cause significant carotid stenosis. 
Carotid intima-medial thickness (CIMT) is defined as the distance between interfaces delineating the luminal aspect of the intimal layer and the outer aspect of the medial layer as seen on carotid ultrasound images. Specifically, the distance is measured between these two white lines seen in the vessel wall when the ultrasound beam is perpendicular to the wall [21]. CIMT is a validated surrogate for cerebrovascular disease [41-43], Specifically, it has been noted that a value greater than or equal to the 75 th percentile of a reference normal population is considered abnormal and indicates an increased risk of CVEs [44]. Lorenz published a meta-analysis in 2007 that correlated CIMT values with the risk of subsequent stroke [37]. He showed that for an absolute increase of CIMT of $0.1 \mathrm{~mm}$, the stroke risk increases by $13-18 \%$.

The use of CIMT has been evaluated as a marker for radiation-induced carotid toxicity. In a 2008 study, radiation effect on the intimamedial thickness was evaluated by Shariat [21]. In an effort to eliminate cofactors for atherosclerosis, the authors excluded patients with risk factors including hypertension, diabetes, connective tissue disorders and prior cerebrovascular accidents. Thirteen patients were evaluated, all of whom had completed radiation to the carotid at least 1 year earlier. The study utilized nonirradiated controls matched for age, sex and race. All radiotherapy patients had received $3 \mathrm{D}$ radiation treatment for nasopharynx cancer. The authors noted that mean CIMT was higher in the irradiated patients than control subjects. This was statistically significant at 0.74 versus $0.46 \mathrm{~mm}$ ( $\mathrm{p}<0.001)$. This correlated with earlier studies in which patients in the irradiated cohort had a higher percentage of abnormal CIMT values that those in the control group [45]. Therefore, the authors concluded that radiation, as the only factor that was different between the two groups, was likely to have contributed to the accelerated rate of atherosclerosis in the irradiated patients.

While multiple trials have evaluated carotid artery IMT in irradiated head and neck cancer patients, many were performed without a baseline study. Therefore, it is not entirely clear whether patients may have suffered from cerebrovascular disease prior to radiation treatment. To eliminate this uncertainty, more recent studies have been performed prospectively while others aim to avoid confounding factors by selecting patients with unilateral radiation treatment utilizing the contralateral un-irradiated carotid as a control. Wilbers performed a prospective study evaluating changes of CIMT in the first 2 years following radiation [46]. All patients underwent neck irradiation for head and neck cancer and CIMT measurement was obtained at baseline and at 2 years after completion of treatment. Patients were followed and evaluated for risk factors and comorbidities. A total of 69 patients was included in the publication. Median CIMT at baseline was $0.60 \mathrm{~mm}$ and at 2 years was 0.62 $\mathrm{mm}$. This change was not statistically significant. The only patient characteristic associated with change in CIMT was patient age. None of the known risk factors that were evaluated, including smoking, hypertension, hypercholesterolemia, obesity or diabetes was related. Median dose delivered to the portion of the carotid at which the CIMT measurement was obtained was 66 Gy. Four of 69 patients experienced stroke or transient ischemic attack (TIA), the mean RT-stroke interval was 6.8 months and the area of CIMT progression was matched to the high-dose radiation port. The authors point out that their study conflicts with previously published studies that did show a CIMT increase in irradiated carotid segments $[47,48]$. However, the time interval in this study was only 2 years. Therefore, longer follow-up may be necessary.

To account for a longer process of evolution of post-irradiation vascular damage, Wilbers evaluated patients prospectively over the first 7 years following radiation treatment to the head/neck area [49]. CIMT was utilized as the method of evaluation and was performed pretreatment and at scheduled intervals. In total, 103 patients were evaluated. Some patients received unilateral irradiation and had evaluation of both the irradiated and unirradiated sides. IMT was performed at the high-dose region as designated by review of the radiation plan. At a median follow-up of 7 years, 51 patients were evaluable. CIMT in irradiated common carotids at baseline was 0.64 and increased to 0.74 . The mean increase of IMT in irradiated and nonirradiated common carotid segments was 0.11 and $0.02 \mathrm{~mm}(\mathrm{p}=0.03)$. CIMT in irradiated internal carotid segments was 0.55 at baseline and 0.59 at follow-up. This change was not statistically significant. Baseline risk factors for atherosclerosis, other than age, were not associated with CIMT increase or with CIMT at follow-up, pointing to radiation treatment as the causative factor. The authors noted that there were 546 person years of data and ten patients suffered a first-ever TIA or stroke. The 
incidence rate was 18 per 1000 person years. The mean time interval to stroke was 3 years and the mean age was 71 years. The authors concluded that increase in CIMT in irradiated carotids was clinically relevant.

\section{Measurement of arterial stiffness}

An alternative to measured change in CIMT as a representation of radiation-induced carotid atherosclerosis is to evaluate arterial stiffness as a biomarker of the damage. In a 2010 publication [50], Vlachopoulos demonstrated significant increases in cardiovascular events and mortality with increases in arterial stiffness. Gujral has published extensively on the topic of radiationinduced carotid injury, including arterial stiffness [51]. A strength of Gujral's study was the selection of patients undergoing unilateral neck irradiation, with the unirradiated side used as a control. All patients in the study had completed treatment at least 2 years prior to evaluation. The study compared the beta stiffness index and elastic modulus via ultrasound to assess for changes in proximal, middle and distal common carotid arteries. A total of 50 patients were evaluated in this way. More than half had received $60 \mathrm{~Gy}$ of radiation to the neck (minimum dose $50 \mathrm{~Gy}$ ), and $68 \%$ also underwent neck dissection. There were no differences in the beta stiffness index, but there was a statistically significant difference in elastic modulus $(\mathrm{p}<0.0001)$ at proximal, middle and distal common carotid artery locations. CIMT was evaluated for each patient. The authors found no difference side to side in patients undergoing surgery. Of note, there were no significant differences in stiffness parameters between the two sides in patients with normal CIMT on the irradiated side. The authors concluded that increased arterial stiffness was not detected before increased CIMT was noted, making it unclear whether arterial stiffness has value as an independent biomarker for arterial toxicity.

\section{Progression of CIMT as an imaging biomarker for cerebrovascular disease}

Gujral's extensive investigation of CIMT as a marker of radiation-induced carotid atherosclerosis has been more fruitful. The same patients in the cohort referenced above were also assessed with CIMT measurements, comparing the irradiated to the unirradiated sides [52]. All patients received at least 50 Gy to unilateral neck volumes. Patients were evaluated for other risks of atherosclerotic disease including diabetes, hypertension, hyperlipidemia, smoking, obesity, history of atrial fibrillation and TIA as well as serum markers. Mean CIMT was obtained for three segments of the carotid as well as at the bulb. The Qstroke score, a validated algorithm used to predict cerebrovascular incidents, was calculated. This algorithm uses clinical risk factors for stroke relating to smoking, diabetes, history of heart disease, obesity and similar factors. Gujral noted that for all four arterial segments measured in irradiated carotids, a significantly greater number of CIMT measurements were above the 75 th percentile of a normal reference population compared with the unirradiated side. None of the other measured factors had an effect and there was no correlation noted between radiation-associated progression of CIMT and Qstroke score, meaning that progression of CIMT with radiation exposure was not confined to patients clinically predisposed to cerebrovascular disease. Gujral concluded that evaluation of changes in CIMT is a relevant way to assess radiation exposure as a factor that can lead to CVEs - a useful imaging biomarker. Further, progression of CIMT caused by radiation is not confined to patients who have pre-existing risk factors for cerebrovascular disease, but instead is a primary independent risk factor for CIMT progression and therefore for an increased risk of CVEs.

\section{Dose-response for radiation-induced carotid toxicity}

As is the case with most somatic effects of radiation on normal tissue, late carotid toxicity is considered a nonstochastic effect with a threshold dose below which no response (biological effect) for a defined end point is seen and above which the incidence/severity of the response is nonlinearly proportional to dose. For carotid stenosis and increased CIMT, Martin et al. [53] evaluated a cohort of 40 patients who underwent unilateral neck irradiation for a variety of malignant diagnoses, including lymphoma, and who were treated with a range of radiation doses. All patients were asymptomatic from a carotid perspective. Ultrasound evaluation showed carotid stenosis on the irradiated side in 14 patients compared with five on the unirradiated side. For stenosis of at least $60 \%$, the hazard ratio for the irradiated side was six. All stenotic lesions on the irradiated side were located within the radiation portals. CIMT was significantly greater on the 
irradiated sides. Most interesting, these indicators of carotid toxicity were confined to the patients who received at least $35 \mathrm{~Gy}$ to the neck (lymphoma patients often receive lower doses). These results were consistent with previously published series showing low rates of carotid stenosis in patients treated for lymphoma $[54,55]$.

\section{Latency of radiation-induced carotid toxicity}

The latency period from the time of irradiation to observed changes in the carotid varies between studies. Cheng conducted a large study examining the carotids with ultrasound in irradiated patients in 1999, with 240 patients evaluated [56]. An interval of greater than 5 years after radiation was significantly associated with a higher risk of internal or common carotid stenosis. In contrast, the cohort studied by Gujral [51] showed no association between postradiation interval and CIMT, despite a mean follow-up of 54 months after radiation treatment.

\section{Computed tomography \&}

contrast-enhanced ultrasound to evaluate radiation-induced carotid toxicity

Another method to evaluate carotid atherosclerosis is with computed tomography (CT) scanning. Anzidei performed a retrospective study examining variations in carotid artery plaque composition and volume using multidetector CT in patients before and 2 years after head and neck irradiation. The study evaluated 62 radiation patients as well as 40 patients treated surgically for head and neck cancer [57]. There was a statistically significant difference in the degree of plaque and stenosis between the two groups, with an increase in the total carotid artery plaque volume, fatty component volume and mixed component volume in irradiated patients. No difference was found in the volume of calcified tissue. Patients treated with surgery alone showed no significant changes in plaque volume or subvolume measures. There was a statistically significant increase in the degree of stenosis noted in both the radiation and surgical groups, but the increase was greater in the radiation group. This study is particularly interesting due to its use of CT to evaluate plaque subcomponents. The strong, statistically significant increase in the fatty component volume suggests that radiation treatment promotes a route of atherosclerosis development via increases in the plaque lipid pool, and it is lipid-rich plaques that are at greatest risk for intra-plaque hemorrhage and rupture.

Gujral provided a recent case report using contrast-enhanced ultrasound to assess plaque neovascularization in irradiated carotid arteries [58]. When ultrasound is used with contrast, it is possible to identify neovascular changes within carotid plaques. These changes are associated with plaque instability [59]. In the case report, the investigators performed a bilateral carotid ultrasound in a patient after unilateral neck irradiation. Carotid plaques were noted on both sides, but the contrasted ultrasound study demonstrated neovascular changes only on the irradiated side, implying an increased risk of stroke on that side compared with the non-irradiated side.

\section{Clinically documented CVEs in irradiated \\ patients}

Clearly, the assessment of imaging biomarkers for radiation-induced carotid toxicity is important to consider. However, it is also important to consider whether the literature documents an excess of clinically documented atherosclerotic events in patients after neck irradiation, including patients with early glottic cancers. In a retrospective study from 2002, Dorresteijn evaluated 367 patients with head and neck tumors of which 162 were T1 or T2 larynx cancers [60]. All patients had received radiation at an age less than 60. Dosing and field size were typical of the era, with 3D plans of 2-2.4 Gy per day to a total dose of 60-66 Gy. The median follow-up was 7.7 years. Overall, the relative risk (RR) of stroke was 5.6 for the irradiated patients compared with a population from a stroke registry matched for age and sex. Median age at stroke was 62.7 years with a median interval from radiation treatment to development of stroke was 10.9 years. Patients with early larynx cancer were assessed specifically and the RR of stroke in this group was 5.1. As parotid patients receive ipsilateral irradiation, these patients were analyzed specifically. There were six strokes in parotid patients, and five occurred on the irradiated side. The 15-year cumulative risk of stroke after neck irradiation was $12.0 \%$. This study was one of the first to show an increased risk of ischemic stroke after radiation to the neck. With regard to timing of carotid toxicity, the Dorresteijn data demonstrated an RR for stroke of 3.7 at less than 10 years from radiation treatment as opposed to 10.1 at more than 10 years. A potential weakness 
of this study is the fact that the control population was not matched specifically for risk factors for cerebrovascular disease.

\section{CVEs in patients with early glottic cancer} after radiation treatment

Several studies have evaluated risk of CVEs specifically in the population that is the subject of this review. Hong focused on patients with early-stage glottic cancer to correlate their risk of stroke with treatment type [61]. The patients were all aged over 65 years and were diagnosed with early-stage glottic cancer between 1992 and 2007 as identified in the SEER database. The authors analyzed records of 1413 patients, of whom 1055 were treated with radiotherapy while 358 were treated surgically. Because the SEER database does not have information on typical cerebrovascular risk factors, the incidence of peripheral vascular events was used as a proxy for these factors. The analysis showed that the risk for CVEs in the irradiated group was not statistically greater than in the surgical group. The 10-year actuarial rate of cerebrovascular disease was $56.5 \%$ in the irradiated patients versus $48.7 \%$ in the operated patients $(p=0.27)$. The hazard ratio for CVD risk was $1.11(\mathrm{p}=0.31)$. Age and more recent year of diagnosis were associated with statistically higher CVD rates. Rates of peripheral vascular disease were the same in both groups, implying similar underlying CVD risk factors. The authors noted that the age of the population in this SEER analysis did not overlap with the population in the Dorresteijn study, so that the results could not be compared directly. They did conclude that the SEER data support their hypothesis that radiotherapy as delivered to the carotids with typical opposed lateral radiation fields for early glottic cancer may cause less carotid toxicity than the longer segments treated for other indications. They note that any conclusions should only be applied to older patients.

Data published at a similar time by SwisherMcClure also specifically evaluated risk of fatal ischemic stroke after radiotherapy for early-stage glottic larynx cancer [62]. SEER data were also utilized in this study. All patients had stage I disease. A total of 7237 patients was identified to have had radiotherapy while 1484 patients underwent surgical management. The specific primary end point was death from ischemic stroke. Multivariable competing risks regression models were used to adjust for potential confounders. Median follow-up was 5.3 years and both groups were similar with regards to patient characteristics. In a multivariate analysis, radiotherapy was associated with an increased risk of fatal stroke compared with surgery (HR: 1.75; $\mathrm{p}=0.04)$. However, it should be noted that the risk of fatal ischemic stroke as related to radiation was $1.3 \%$ at 15 years, resulting in a number needed to harm of 77 . In other words, the data showed that there would be one excess death due to stroke for every 77 patients treated with radiotherapy for early glottic cancer. There was no difference in overall survival between the groups. The authors noted that SEER has data only on fatal strokes, which comprise about $20 \%$ of all strokes, so a SEER analysis will underestimate the burden of cerebrovascular disease on the studied population. For this reason, it is possible that the increased impact of cerebrovascular toxicity for the irradiated patients compared with the surgical patients in the Swisher-McClure study may be five-times as great as documented.

Management of radiation-induced carotid stenosis

It is inevitable that some patients will develop symptoms of ischemic carotid disease after neck irradiation and will require intervention with carotid stenting or endarterectomy. Sano published a study examining the relationship between radiologic and histologic features in symptomatic radiation-induced carotid stenosis patients and outcomes of carotid revascularization [63]. The authors evaluated 17 patients between July 2004 and August 2014. Among the group, there were 22 carotid revascularizations. The control group was 475 patients without a history of radiotherapy who underwent carotid revascularization during the same time period. All patients had pre-operative imaging followed by either carotid endarterectomy or carotid artery stenting. The resected plaques were examined histologically. Mean time at revascularization was 16.9 years after radiotherapy. Carotid risk factors were more favorable overall in the irradiated group. Pre-operative imaging showed a slightly lower degree of stenosis in the irradiated group, but higher rates of vulnerable plaques, mobile plaques, ulcerative plaques and an especially notable increased rate of multiple ulcerative plaques in the irradiated group. These findings were confirmed after endarterectomy, with significantly higher rates of necrotic plaques and vulnerable, mobile and ulcerative plaques seen in the irradiated group. Over the years, 
there has been concern for performing endarterectomy in a previously irradiated patient due to risk of complication. However, clinical outcomes in this study for stented irradiated patients were inferior to stented controls, while outcomes of operated irradiated patients were the same as operated controls. The authors concluded that, due to higher risk of vulnerable plaque and risk of micro-embolism during stenting, that stent risk may be elevated in irradiated patients and that endarterectomy should be considered for this group.

\section{IMRT for early glottic cancers: the controversy}

The above discussion highlights the merits of IMRT in sparing the carotid arteries along with the concerns voiced against its widespread acceptance as a new standard of treatment. The following is a brief assessment of some of the issues discussed:

- The entire larynx is targeted with 3DCRT, minimizing the possibility of a geographic miss. The margin around the involved vocal $\operatorname{cord}(s)$ is so generous that the technique has empirically developed built-in safeguards against interfraction and intrafraction organ motion and there is little concern about precision of treatment delivery. Rates of local tumor control with 3DCRT are high and wellestablished. Conversely, IMRT offers tailored treatment that follows modern principles of radiotherapy including inverse planning to reduce dose to normal structures at risk (e.g., carotid system), creating a target volume that can take into account organ motion and that allows for more accurate and precise control of dose distribution and dose delivery. This minimizes treatment of neck tissues distant from the actual tumor;

- The argument is often made that data documenting a direct link between radiationinduced carotid arterial toxicity specifically from radiation treatment of early glottic cancers are scarce. There is no doubt that IMRT can substantially reduce the dose to the carotid arteries when treating early glottic cancers in comparison with 3DCRT. There is also clear evidence that, in general, irradiation of the neck is associated with an increased risk of carotid arterial stenosis, atherosclerotic plaque formation and increased carotid intima-media thickness. Studies have shown a statistically significant association between neck irradiation and the incidence of CVE. Moreover, the segment of the carotid system irradiated in early laryngeal cancers usually includes the carotid bulbs and the distal common carotid arteries, segments that may be of vital clinical relevance with respect to atheromatous plaque formation. Thus, although published works showing an increase in IMT with neck irradiation include patients in whom considerable lengths of the carotids were irradiated (from several centimeters below to several centimeters above the carotid bulb), the segments irradiated in early glottis cancers were still encompassed and evaluated in these studies.

\section{Conclusion: a new standard of care?}

The above review leaves no doubt as to the ability of IMRT to reduce substantially carotid radiation doses compared with 3DCRT in the treatment of early glottic cancers. Based on the literature reviewed, there is clear evidence that high-dose carotid irradiation is associated with an increased risk of several aspects of carotid arterial toxicity (stenosis, increase in intimamedia thickness, increased arterial wall stiffness and accelerated progression of atherosclerotic plaque, including the most unstable and dangerous plaque subtypes). These end points are all known predictors for CVEs. Further, we have presented studies that move beyond surrogate end points and show statistically significant association between neck irradiation and increased incidence of CVEs, the ultimate end point for carotid toxicity.

The question, therefore, is whether the very plausible advantage of carotid sparing of radiation dose outweighs the hypothetical risk of local tumor recurrence due to organ motion and the decreased dosimetric margin in IMRT compared with 3DCRT. But when the local control data from the two techniques are reviewed, there is no hint of an increase in local recurrence with IMRT. These data parallel local control data for IMRT compared with 3DCRT in other head/neck tumor sites.

Standardization of IMRT planning for early glottic cancers including target volumes and carotid dose constraints is clearly desirable. The data using both surrogate markers for and direct evidence of carotid toxicity suffices as evidence, in our view, for the importance of avoidance of the carotid arteries when irradiating the glottis. Thus, it is our conclusion that high-quality 


\section{EXECUTIVE SUMMARY}

\section{Development of intensity-modulated radiation therapy}

- Developed in the 1980 s and 1990s and implemented widely since 2000.

- Allows the delivery of complex radiation dose distributions with concave shapes that avoid sensitive normal structures near tumor targets.

- Widely accepted for all head/neck radiation treatment situations due to sparing of salivary, neural and other structures.

- Treatment of early glottic larynx cancers with intensity-modulated radiotherapy (IMRT) remains controversial.

\section{Conventional radiotherapy for early glottic cancer}

- Standard of care since the 1950s with very simple planning and delivery.

- High rate of local control and low rate of acute toxicity.

- In contrast to IMRT, delivers the full tumor dose to the carotid arteries through incidental exposure.

Feasibility \& results of IMRT for early glottic cancer

- Excellent carotid sparing from unintended radiation dose.

- Rapid planning and delivery using current technology.

- Equivalent local control to conventional radiotherapy.

Radiation-induced carotid injury: useful biomarkers

- Measurement of carotid intima-medial thickness (CIMT) is the most widely accepted technique to assess radiationinduced carotid injury.

- High CIMT values are associated with an excess of cerebrovascular events (CVEs).

- Longitudinal data show significant progression of CIMT after radiation exposure to the carotids.

- Unilateral neck radiation studies show progression of CIMT on the irradiated side compared with the untreated side.

- CIMT progression is not confined to patients with pre-existing risk factors for CVE, but is associated independently with radiation treatment.

- Arterial stiffness may not have value as an imaging biomarker independent of CIMT.

- Computed tomography and contrasted ultrasound show significant correlation between carotid plaque volume, morphology, subcomponent distribution and neovascularization (high-risk features) and carotid radiation exposure.

Radiation-induced carotid injury: additional parameters

- The threshold for carotid radiation injury appears to be approximately 35 Gy total dose.

- Data describing the latency of carotid radiation injury are inconsistent, but it appears that risk is significantly greater after 10 years from exposure compared with before 10 years.

\section{Clinically documented CVEs in irradiated patients}

- There is a significant excess rate of CVE in patients with a history of neck radiation including the carotids.

- The preponderance of strokes in patients irradiated to one side of the neck correlate with the treated side.

- Data correlating increased CVE specifically with treatment of early glottic cancers (relatively short carotid segments) include only two SEER analyses - one negative and one positive.

\section{Management of radiation-induced carotid stenosis}

- Carotid stenting may carry a higher risk in irradiated patients than in others.

- Carotid endarterectomy may be the preferred procedure.

\section{Conclusion}

- The data for radiation-induced carotid injury at clinically relevant doses is compelling, with no conceptual reason to expect that treatment of early glottic cancer is an exception.

- There is no technical disadvantage to the use of IMRT for early glottic cancers.

- Radiation oncologists should transition to IMRT as a new standard of care for early glottic cancers. 
IMRT should be adopted as a new standard of care for the treatment of early glottic cancers.

\section{Future perspective}

We would prefer that this issue be resolved by a randomized trial of 3DCRT versus IMRT. However, no trial of this type has been conducted and it is unlikely that such a trial will materialize in the near future. Rather than await randomized controlled data, IMRT for all other head/neck cancers, it should be remembered, has been accepted universally due its clear superiority in reducing doses to normal structures without a reduction in local tumor control. The reason why early glottic cancers should be an exception is unclear. Without a significant technical barrier precluding the use of IMRT for these patients, we believe that it is time for the practice to be adopted widely. There is little doubt that the next decade will see this transition come to pass. Preliminary data on intensity-modulated SVCI are promising. Five to 10 years from now, it is likely that radiation oncologists will be debating which patients are optimal for SVCI, not about whether to use IMRT to treat early larynx cancer.

\section{Financial \& competing interests disclosure}

The authors have no relevant affiliations or financial involvement with any organization or entity with a financial interest in or financial conflict with the subject matter or materials discussed in the manuscript. This includes employment, consultancies, honoraria, stock ownership or options, expert testimony, grants or patents received or pending, or royalties.

No writing assistance was utilized in the production of this manuscript.

\section{Open access}

This work is licensed under the Creative Commons Attribution-NonCommercial 3.0 Unported License. To view a copy of this license, visit http://creativecommons.org/ licenses/by-nc-nd/3.0/

\section{References}

Papers of special note have been highlighted as: - of interest; $\bullet$ of considerable interest

1 Webb S. The physical basis of IMRT and inverse planning. Br. J. Radiol. 76(910), 678-689 (2003).

-. An excellent overview of the history and development of intensity-modulated radiotherapy (IMRT).

2 O'Sullivan B, Rumble RB, Warde P; Members of the IMRT Indications Expert Panel. Intensity-modulated radiotherapy in the treatment of head and neck cancer. Clin. Oncol. (R. Coll. Radiol.) 24(7), 474-487 (2012).

3 Chao KS, Majhail N, Huang CJ et al. Intensity-modulated radiation therapy reduces late salivary toxicity without compromising tumor control in patients with oropharyngeal carcinoma: a comparison with conventional techniques. Radiother. Oncol. 61(3), 275-280 (2001).

4 Li Y, Taylor JM, Ten Haken RK, Eisbruch A. The impact of dose on parotid salivary recovery in head and neck cancer patients treated with radiation therapy. Int. J. Radiat. Oncol. Biol. Phys. 67(3), 660-669 (2007).

5 Setton J, Caria N, Romanyshyn J et al. Intensity-modulated radiotherapy in the treatment of oropharyngeal cancer: an update of the Memorial Sloan-Kettering Cancer Center experience. Int. J. Radiat. Oncol. Biol. Phys. 82(1), 291-298 (2012).
6 Zhang X, Liu F, Lan X et al. Clinical observation of submandibular gland transfer for the prevention of xerostomia after radiotherapy for nasopharyngeal carcinoma: a prospective randomized controlled study of 32 cases. Radiat. Oncol. 9, 62 (2014).

7 Smith RV, Kotz T, Beitler JJ, Wadler S. Long-term swallowing problems after organ preservation therapy with concomitant radiation therapy and intravenous hydroxyurea: initial results. Arch. Otolaryngol. Head Neck Surg. 126(3), 384-389 (2000).

8 Lambade PN, Lambade D, Goel M. Osteoradionecrosis of the mandible: a review. Oral Maxillofac. Surg. 17(4), 243-249 (2013).

9 Mohan R. Field shaping for threedimensional conformal radiation therapy and multileaf collimation. Semin. Radiat. Oncol. 5(2), 86-99 (1995).

10 Kerr P, Myers CL, Butler J, Alessa M, Lambert P, Cooke AL. Prospective functional outcomes in sequential population based cohorts of stage III/IV oropharyngeal carcinoma patients treated with $3 \mathrm{D}$ conformal vs. intensity modulated radiotherapy. J. Otolaryngol. Head Neck Surg. 44: 17 (2015).

11 Broglie MA, Soltermann A, Haile SR et al. Quality of life of oropharyngeal cancer patients with respect to treatment strategy and p16-positivity. Laryngoscope 123(1), 164-170 (2013).

12 Mourad WF, Hu KS, Shourbaji RA et al. Exploration of the role of radiotherapy in the management of early glottic cancer with complete carotid artery occlusion. Onkologie 36(7-8), 433-435 (2013).

13 Chera BS, Amdur RJ, Morris CG, Mendenhall WM. Carotid-sparing intensitymodulated radiotherapy for early-stage squamous cell carcinoma of the true vocal cord. Int. J. Radiat. Oncol. Biol. Phys. 77(5), 1380-1385 (2010).

14 Chatani M, Matayoski Y, Masaki N, Teshima $\mathrm{T}$, Inoue $\mathrm{T}$. Radiation therapy for early glottic carcinoma (T1N0M0). The final results of prospective randomized study concerning radiation field. Strablenther. Onkol. 172(3), 169-172 (1996).

15 Mendenhall WM, Parsons JT, Million RR, Fletcher GH. T1-T2 squamous cell carcinoma of the glottic larynx treated with radiation therapy: relationship of dosefractionation factors to local control and complications. Int. J. Radiat. Oncol. Biol. Phys. 15(6), 1267-1273 (1988).

- Significant publication reviewing the University of Florida experience with conventional radiotherapy for early glottic cancer, with an excellent discussion of radiation dose and fractionation.

16 Fein DA, Mendenhall WM, Parsons JT, Million RR. T1-T2 squamous cell carcinoma of the glottic larynx treated with radiotherapy: a multivariate analysis of variables potentially influencing local control. Int. J. Radiat. Oncol. Biol. Phys. 25(4), 605-611 (1993). 
17 Mendenhall WM, Amdur RJ, Morris CG, Hinerman RW. T1-T2N0 squamous cell carcinoma of the glottic larynx treated with radiation therapy. J. Clin. Oncol. 19(20), 4029-4036 (2001).

18 Schwaibold F, Scariato A, Nunno M et al. The effect of fraction size on control of early glottic cancer. Int. J. Radiat. Oncol. Biol. Phys. 14(3), 451-454 (1988).

19 Al-Mamgani A, van Rooij PH, Woutersen DP et al. Radiotherapy for T1-2N0 glottic cancer: a multivariate analysis of predictive factors for the long-term outcome in 1050 patients and a prospective assessment of quality of life and voice handicap index in a subset of 233 patients. Clin. Otolaryngol. 38(4), 306-312 (2013).

- The largest published series of conventionally treated early larynx patients with an analysis of voice outcomes.

20 Yamazaki H, Nishiyama K, Tanaka E, Koizumi M, Chatani M. Radiotherapy for early glottic carcinoma (T1N0M0): results of prospective randomized study of radiation fraction size and overall treatment time. Int. J. Radiat. Oncol. Biol. Phys. 64(1), 77-82 (2006).

-• A landmark randomized trial demonstrating improved outcomes with hypofractionated radiotherapy compared with standard fractionation for early glottic cancer.

21 Shariat M, Alias NA, Biswal BM. Radiation effects on the intima-media thickness of the common carotid artery in post-radiotherapy patients with head and neck malignancy. Postgrad. Med. J. 84(997), 609-612 (2008).

22 Osman SO, Astreinidou E, de Boer $\mathrm{HC}$ et al. IMRT for image-guided single vocal cord irradiation. Int. J. Radiat. Oncol. Biol. Phys. 82(2), 989-997 (2012).

23 Osman SO, Astreinidou E, Levendag PC, Heijmen BJ. Impact of geometric variations on delivered dose in highly focused single vocal cord IMRT. Acta Oncol. 53(2), 278-285 (2014).

24 Riegel AC, Antone J, Schwartz DL. Comparative dosimetry of volumetric modulated arc therapy and limited-angle static intensity-modulated radiation therapy for early-stage larynx cancer. Med. Dosim. 38(1), 66-69 (2013).

25 Matthiesen C, Herman Tde L, Singh H et al. Dosimetric and radiobiologic comparison of 3D conformal, IMRT, VMAT and proton therapy for the treatment of early-stage glottic cancer. J. Med. Imaging Radiat. Oncol. 59(2), 221-228 (2015).

- One of the only published studies evaluating proton radiation therapy for early glottic cancers, in this paper in the context of several other techniques.

26 Zumsteg ZS, Riaz N, Jaffery $S$ et al. Carotid sparing intensity-modulated radiation therapy achieves comparable locoregional control to conventional radiotherapy in $\mathrm{T} 1-2 \mathrm{~N} 0$ laryngeal carcinoma. Oral Oncol. 51(7), 716-723 (2015).

27 Feigenberg SJ, Lango M, Nicolaou N, Ridge JA. Intensity-modulated radiotherapy for early larynx cancer: is there a role? Int. J. Radiat. Oncol. Biol. Phys. 68(1), 2-3 (2007).

28 Vanetti E, Clivio A, Nicolini G et al. Volumetric modulated arc radiotherapy for carcinomas of the oro-pharynx, hypopharynx and larynx: a treatment planning comparison with fixed field IMRT. Radiother. Oncol. 92(1), 111-117 (2009).

29 Broggi S, Perna L, Bonsignore F et al. Static and rotational intensity modulated techniques for head-neck cancer radiotherapy: a planning comparison. Phys. Med. 30 (8), 973-979 (2014).

30 Kubicek GJ, Machtay M. New advances in high-technology radiotherapy for head and neck cancer. Hematol. Oncol. Clin. North Am. 22(6), 1165-1180, viii (2008).

31 Penagaricano JA, Ratanatharathorn V, Papanikolaou N, Yan Y. Intensity-modulated radiation therapy reduces the dose to normal tissue in T2N0M0 squamous cell carcinoma of the glottic larynx. Med. Dosim. 29(4), 254-257 (2004).

32 Rosenthal DI, Fuller CD, Barker JL Jr et al. Simple carotid-sparing intensity-modulated radiotherapy technique and preliminary experience for T1-2 glottic cancer. Int. J. Radiat. Oncol. Biol. Phys. 77(2), 455-461 (2010).

33 Berwouts D, Swimberghe M, Duprez F et al. Intensity-modulated radiotherapy for early-stage glottic cancer. Head Neck 38(Suppl. 1), E179-E184 (2016).

34 Lambrecht M, Nevens D, Nuyts S. Intensitymodulated radiotherapy vs. parotid-sparing 3D conformal radiotherapy. Effect on outcome and toxicity in locally advanced head and neck cancer. Strablenther. Onkol. 189(3), 223-229 (2013).

35 Eisbruch A, Harris J, Garden AS et al. Multi-institutional trial of accelerated hypofractionated intensity-modulated radiation therapy for early-stage oropharyngeal cancer (RTOG 00-22). Int. J. Radiat. Oncol. Biol. Phys. 76(5), 1333-1338 (2010).

36 Al-Mamgani A, Kwa SL, Tans L et al. Single vocal cord irradiation: image guided intensity modulated hypofractionated radiation therapy for T1a glottic cancer: early clinical results. Int. J. Radiat. Oncol. Biol. Phys. 93(2), 337-343 (2015).

37 Lorenz MW, Markus HS, Bots ML, Rosvall M, Sitzer M. Prediction of clinical cardiovascular events with carotid intimamedia thickness: a systematic review and meta-analysis. Circulation 115(4), 459-467 (2007).

38 Chambless LE, Folsom AR, Clegg LX et al. Carotid wall thickness is predictive of incident clinical stroke: the Atherosclerosis Risk in Communities (ARIC) study. Am. J. Epidemiol. 151(5), 478-487 (2000).

39 Plummer C, Henderson RD, O'Sullivan JD, Read SJ. Ischemic stroke and transient ischemic attack after head and neck radiotherapy: a review. Stroke 42(9), 2410-2418 (2011).

40 Lam WW, Leung SF, So NM et al. Incidence of carotid stenosis in nasopharyngeal carcinoma patients after radiotherapy. Cancer 92(9), 2357-2363 (2001).

41 Bots ML, Groenewegen KA, Anderson TJ et al. Common carotid intima-media thickness measurements do not improve cardiovascular risk prediction in individuals with elevated blood pressure: the USE-IMT collaboration. Hypertension 63(6), 1173-1181 (2014).

42 Hollander M, Hak AE, Koudstaal PJ et al. Comparison between measures of atherosclerosis and risk of stroke: the Rotterdam Study. Stroke 34(10), 2367-2372 (2003).

43 Ali YS, Rembold KE, Weaver B et al. Prediction of major adverse cardiovascular events by age-normalized carotid intimal medial thickness. Atherosclerosis 187(1), 186-190 (2006).

44 Aminbakhsh A, Mancini GB. Carotid intimamedia thickness measurements: what defines an abnormality? A systematic review. Clin. Invest. Med. 22(4), 149-157 (1999).

45 So NM, Lam WW, Chook P, Woo KS, Liu $\mathrm{KH}$, Leung SF et al. Carotid intima-media thickness in patients with head and neck irradiation for the treatment of nasopharyngeal carcinoma. Clin. Radiol. 57(7), 600-603 (2002)

46 Wilbers J, Hoebers FJ, Boogerd W et al. Prospective cohort study of carotid intimamedia thickness after irradiation. J. Stroke Cerebrovasc. Dis. 23(10), 2701-2707 (2014).

47 Muzaffar K, Collins SL, Labropoulos N, Baker WH. A prospective study of the effects of irradiation on the carotid artery. Laryngoscope 110(11), 1811-1814 (2000). 
48 Pereira Lima MN, Biolo A, Foppa M, da Rosa PR, Rohde LE, Clausell N. A prospective, comparative study on the early effects of local and remote radiation therapy on carotid intima-media thickness and vascular cellular adhesion molecule-1 in patients with head and neck and prostate tumors. Radiother. Oncol. 101(3), 449-453 (2011).

49 Wilbers J, Dorresteijn LD, Haast R et al. Progression of carotid intima media thickness after radiotherapy: a long-term prospective cohort study. Radiother. Oncol. 113(3), 359-363 (2014).

-• An excellent long-term examination of the effect of radiotherapy on carotid stenosis.

50 Vlachopoulos C, Aznaouridis K, Stefanadis C. Prediction of cardiovascular events and all-cause mortality with arterial stiffness: a systematic review and meta-analysis. J. Am. Coll. Cardiol. 55(13), 1318-1327 (2010).

51 Gujral DM, Shah BN, Chahal NS et al. Arterial stiffness as a biomarker of radiationinduced carotid atherosclerosis. Angiology 67(3), 266-271 (2016).

52 Gujral DM, Shah BN, Chahal NS et al. Carotid intima-medial thickness as a marker of radiation-induced carotid atherosclerosis. Radiother. Oncol. 118(2), 323-329 (2016).

-. The most detailed examination in the literature of all aspects of the effects of radiotherapy on the carotid intima-media complex.

53 Martin JD, Buckley AR, Graeb D, Walman B, Salvian A, Hay JH. Carotid artery stenosis in asymptomatic patients who have received unilateral head-and-neck irradiation. Int. J. Radiat. Oncol. Biol. Phys. 63(4), 1197-1205 (2005).

54 King LJ, Hasnain SN, Webb JA et al. Asymptomatic carotid arterial disease in young patients following neck radiation therapy for Hodgkin lymphoma. Radiology 213(1), 167-172 (1999).

55 Dubec JJ, Munk PL, Tsang V et al. Carotid artery stenosis in patients who have undergone radiation therapy for head and neck malignancy. Br. J. Radiol. 71(848), 872-875 (1998).

56 Cheng SW, Wu LL, Ting AC, Lau H, Lam LK, Wei WI. Irradiation-induced extracranial carotid stenosis in patients with head and neck malignancies. Am. J. Surg. 178(4), 323-328 (1999).

57 Anzidei M, Suri JS, Saba L, Sanfilippo R, Laddeo G, Montisci R et al. Longitudinal assessment of carotid atherosclerosis after radiation therapy using computed tomography: a case control Study. Eur. Radiol. 26(1), 72-78 (2016).

58 Gujral DM, Shah BN, Bhattacharyya S, Senior R, Harrington KJ, Nutting CM.
Contrast-enhanced ultrasound to assess plaque neovascularization in irradiated carotid arteries. Int. J. Cardiol. 202, 3-4 (2016).

59 Koole D, Heyligers J, Moll FL, Pasterkamp G. Intraplaque neovascularization and hemorrhage: markers for cardiovascular risk stratification and therapeutic monitoring. J. Cardiovasc. Med. (Hagerstown) 13(10), 635-639 (2012).

60 Dorresteijn LD, Kappelle AC, Boogerd W et $a l$. Increased risk of ischemic stroke after radiotherapy on the neck in patients younger than 60 years. J. Clin. Oncol. 20(1), 282-288 (2002).

- One of the few published studies directly linking carotid irradiation to stroke.

61 Hong JC, Kruser TJ, Gondi V et al. Risk of cerebrovascular events in elderly patients after radiation therapy versus surgery for early-stage glottic cancer. Int. J. Radiat. Oncol. Biol. Phys. 87(2), 290-296 (2013).

62 Swisher-McClure S, Mitra N, Lin A et al. Risk of fatal cerebrovascular accidents after external beam radiation therapy for early-stage glottic laryngeal cancer. Head Neck 36(5), 611-616 (2014).

63 Sano N, Satow T, Maruyama D et al. Relationship between histologic features and outcomes of carotid revascularization for radiation-induced stenosis. J. Vasc. Surg. 62(2), 370-377.e1 (2015). 